\title{
FORMAL PROPERTIES OF RULE ORDERINGS IN LINGUISTICS
}

\author{
Francis Jeffry Pelletier \\ Dept. Philosophy, Univ. Alberta, Edmonton Alberta Canada
}

\section{Abstract}

The discovery in the late 1960's that standard linguistic theory (of Chomsky's Aspects) was equivalent in generative power to unrestricted rewrite rules caused linguists to search for a "stronger linguistic metatheory". It seemed to some of these researchers that this meant describing linguistic theory by means of rules which were more restricted than type 0 languages. Such a view we call the L-view of constraints on linguistic theory: it advocates constraining the allowable rules in such a way that legitimate grammars can no longer generate arbitrary r.e. sets, but only some subset of them. To other researchers this discovery meant rather that one should place restrictions on linguistic theory so that the kinds of grammars allowed would be limited, regardless of whether such limitations affected the generative power of the theory. We call this the G-view of constraints. The $L-$ and $G$-views are not equivalent limitations. For example, a G-view limitation on the class of regular grammars that any legitimate grammar be right-embedding is not the reby a L-view limitation, since this does not effect an alteration in generative power of the grammars allowed. The G-view is avowedly psychological; according to it, the point of placing constraints on grammars is to lessen directly the language learner's burden of choosing the correct grammar from all the possible ones. For the L-view, this is a side effect of disallowing whole classes of grammars in the first place.

One area in linguistics where restrictions on linguistic theory have been advocated is in the ordering (within the cycle) of the application of the rules which generate the language. Here are eight proposals that have been aired in linguistics about how the rules might be ordered.

Total Ordering: the re is a unique first rule, a unique second, .... a unique last rule. Every derivation applies the rules in this order.

Partial Ordering: there is a unique first rule, but thereafter at every stage of a derivation there are two rules which are candidates for application: the rule which was just applied and the (unique) next different rule.
Semi Ordering: the rules are given a total ordering, but different derivations may start at different places in the ordering and choose any "later" rule as the next rule to be applied.

Semi Partial Ordering: the rules are given a partial ordering, but different derivations may start at different places in the ordering and choose either the last-applied rule or else any "later" rule as the next rule to be applied.

Unorderings: any derivation can apply the rules in any order, subject only to the constraint that once a rule has been applied in a derivation, it is no longer eligible for application at a later stage of that derivation.

Quasi Ordering: any derivation can apply the rules in any order, subject only to the constraint that once a rule has been applied in a derivation, the only other time it may be applied in that derivation is to its own output.

Random Ordering: there is no order imposed on the rules; any derivation can apply the rules in any order.

Simultaneous Application: the entire set of rules is applied to an input "all at once"; this prevents some of the rules from creating or destroying part of the input in such a way as to affect the applicability of other rules.

Advocates of one or another of these rule orderings have adopted the G-view, and feel that the rule ordering restrictions they desire will directly lessen the psychological task of learning the correct grammar. The arguments for this conclusion are canvassed and found to be inconclusive (at best). The present paper adopts the L-view and, consequently, investigates the issue of generative power of the various theories of rule orderings. We are really interested in more than just the weak generative power of these theories: we would want to know whether every empirical claim (such as ambiguity, paraphrase, etc.) which can be made by one theory could also be made by another (their strong generative capacity). We trust that if, for any grammar obeying rule ordering conditions $A$ there is a grammar obeying rule ordering conditions $B$ such that they both obey standard linguistic strictures 
on the correctness of rules and such that the two contain exactly the same class of derivations, then rule ordering theory $B$ is at least as powerful in strong generative capacity as rule ordering theory $A$. If the converse can also be established, then they are equivalent in strong generative capacity; if the converse cannot also be established then rule ordering theory $B$ is more powerful in strong generative capacity than rule ordering theory $A$. If they can each be shown to generate empirical claims the other can't, then they are non-comparable in strong generative capacity. A series of theorems are proved resulting in the relationships illustrated in Figure 1 (attached).

Some linguists who advocate "random ordering" actually have in mind random ordering plus some "universal principles". These universal principles are intended to rule out certain derivations - - those derivations in which pairs of rules are not applied in the order specified by the principles. (Also, since their proponents hold the G-view, these principles are supposed to correspond to psychologically plausible learning/processing strategies). We investigate the effect of four of these principles from the standpoint of the L-view, showing that two of them are strongly equivalent to total orderings and that two of them are intermediate between total and partial orderings.

We close with an indication of what the role of mathematical linguistics should be for the ordinary working linguist.

\section{RANDOM ORDERINGS}<smiles>c1ccccc1</smiles>

QUASI ORDERINGS

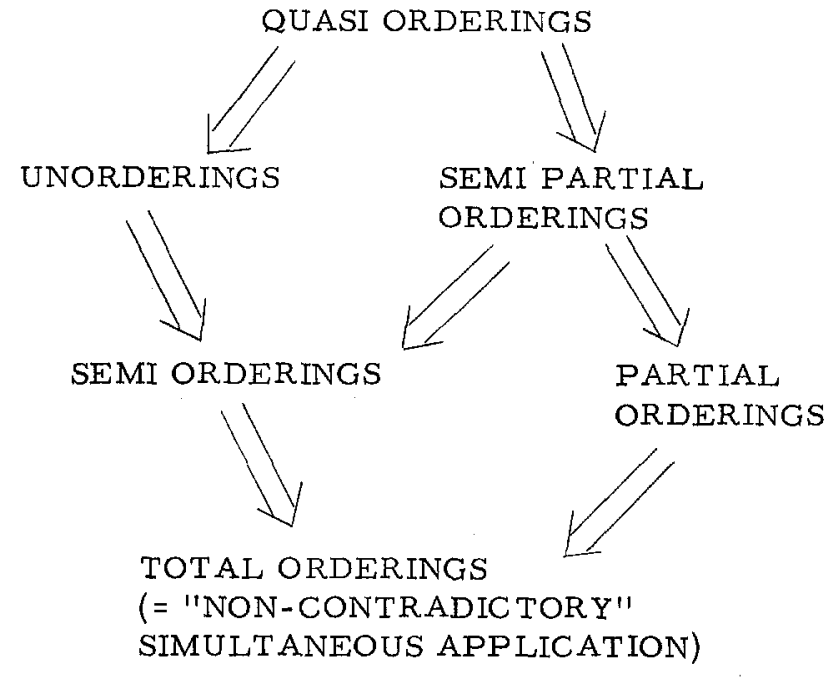

FIGURE 1: Relative Strong Generative Capacities of Various Theories of Rule Orderings. $X \Rightarrow Y$ means that theory $X$ is stronger than theory $\mathrm{Y} .(\Longrightarrow$ is transitive). 